\title{
UTILIZAÇÃO DO FACTSAGE NO ESTUDO DA DESSILICIAÇÃO DO FERRO-GUSA*
}

\author{
Diego Coradini ${ }^{1}$ \\ Heitor Cristo Clem de Oliveira ${ }^{2}$ \\ Anna Paula Littig Berger ${ }^{3}$ \\ Raphael de Alcantara Sampaio ${ }^{2}$ \\ Romulo Camata ${ }^{4}$ \\ José Roberto de Oliveira ${ }^{5}$
}

\section{Resumo}

O objetivo deste trabalho é estudar as reações de dessiliciação, envolvidas no processo de pré-tratamento de ferro-gusa. A princípio, as misturas utilizadas foram caracterizadas e estudadas através do software de termodinâmica computacional (FactSage). Em seguida, os resultados obtidos através do estudo termodinâmico computacional foram comparados com aqueles obtidos mediante os testes experimentais a $1400{ }^{\circ} \mathrm{C}$. As misturas dessiliciantes tiveram como base fontes de $\mathrm{CaO}$ e $\mathrm{FeO}$. Os testes experimentais permitiram identificar que a agitação do sistema, maior quantidade de massa de mistura adicionada e misturas com adição de $20 \%$ de fonte de $\mathrm{CaO}$ mostraram-se mais eficazes no processo de dessiliciação do ferro-gusa. Com os resultados pode-se afirmar que a termodinâmica computacional mostrou-se eficaz em prever o processo. Além disso, a termodinâmica computacional também contribui, em alguns casos, para a análise de compostos sólidos que podem se formar na escória e limitar as reações de dessiliciação.

Palavras-chave:Dessiliciação; Termodinâmica Computacional; Ferro-gusa.

\section{USE OF FACTSAGE IN THE STUDY OF HOT METAL DESILICONIZATION}

\begin{abstract}
The aim of this work is to study the desiliconization reactions involved in the hot metal pretreatment process. At first, the mixtures used were characterized and studied by the computational thermodynamics software, FactSage. Then, the results obtained by this study were compared with those obtained by experimental tests at $1400{ }^{\circ} \mathrm{C}$. The mixtures used in the desiliconization were based on $\mathrm{CaO}$ and $\mathrm{FeO}$. The experiments allowed identifying that, with the use of agitation, higher amount of mixture and mixtures with addition of $20 \%$ of $\mathrm{CaO}$ provoked an increase in the desiliconization efficiency. With the results, it was possible to declare that the computational thermodynamic showed effectiveness to predict the process. Moreover, computational thermodynamic also contributed, in some cases, for analysis of solid compounds that could form in the slag and it could limit the desiliconization reactions, which depends on the mass transport in the metal-slag interface.
\end{abstract}

Keywords: Desiliconization; Computational Thermodynamics; Hot Metal.

1 Engenheiro Metalurgista e Mestrando em Engenharia Metalúrgica e de Materiais, Instituto Federal do Espírito Santo, Vitória, ES, Brasil.

2 Mestre em Engenharia Metalúrgica e de Materiais, Instituto Federal do Espírito Santo, Vitória, ES, Brasil.

3 Engenheira Metalurgista e Mestranda em Engenharia Metalúrgica e de Materiais, Instituto Federal do Espírito Santo, Vitória, ES, Brasil.

4 Graduando em Engenharia Metalúrgica, Instituto Federal do Espírito Santo, Vitória, ES, Brasil.

5 Prof. Dr. do Instituto Federal do Espírito Santo, IFES, dos cursos de graduação e pós-graduação em Engenharia Metalúrgica e de Materiais. 


\section{INTRODUÇÃO}

O silício tem influência significante sobre as demais etapas de pré-tratamento e refino do ferro-gusa, principalmente na desfosforação, uma vez que o mesmo possui grande afinidade pelo oxigênio e, portanto, oxida antes do fósforo. Sendo assim, torna-se necessário a remoção prévia do silício do metal a fim de realizar as demais etapas de refino do aço com maior eficiência e estabilidade.

A diminuição da concentração de silício no ferro-gusa é realizada através da reação de oxidação do mesmo mediante a injeção de uma fonte de oxigênio no metal. $O$ oxigênio pode ser fornecido mediante sopro de oxigênio gasoso ou através de injeção de mistura contendo minério de ferro ou carepa.

A oxidação do silício gera sílica $\left(\mathrm{SiO}_{2}\right)$ que é fixada na escória através do óxido de cálcio $(\mathrm{CaO})$. As reações de oxidação do silício (mediante fonte de oxigênio gasoso e sólido) e fixação da sílica na escória são mostradas através das equações abaixo. $[1,2]$

$$
\begin{array}{r}
{[\mathrm{Si}]+\mathrm{O}_{2(\mathrm{~g})}=\left(\mathrm{SiO}_{2}\right)} \\
\Delta G_{1}^{0}=-821780+221 \mathrm{~T}\left(\frac{\mathrm{J}}{\mathrm{mol}}\right) \\
{[\mathrm{Si}]+2(\mathrm{FeO})=\left(\mathrm{SiO}_{2}\right)+2[\mathrm{Fe}]} \\
\Delta G_{3}^{0}=-356020+130,47 \mathrm{~T}\left(\frac{\mathrm{J}}{\mathrm{mol}}\right) \\
{[\mathrm{Si}]+2(\mathrm{FeO})+3(\mathrm{CaO})=\left(3 \mathrm{CaO} . \mathrm{SiO}_{2}\right)+2[\mathrm{Fe}]} \\
\Delta G_{5}^{0}=-128400-1,9 T\left(\frac{\mathrm{J}}{\mathrm{mol}}\right)
\end{array}
$$

Yamagata et al [3] estudaram a reação de dessiliciação utilizando escória de alto forno e avaliaram o efeito da basicidade da escória sobre tal processo. Segundo os autores o aumento da basicidade da escória (aumento da concentração de $\mathrm{CaO}$ e diminuição da concentração de $\mathrm{SiO}_{2}$ ) contribui para o aumento da redução de silício do ferro-gusa. Sendo assim, quando o ferro-gusa possui concentração de silício elevada, é viável realizar o seu pré-tratamento a fim de otimizar as etapas subsequentes.

Além de contribuir para a otimização e estabilização nos processos de desfosforação e refino no convertedor LD, a dessiliciação prévia do ferro-gusa ainda traz como benefícios a diminuição no consumo de $\mathrm{CaO}$ e volume de escória gerada no convertedor, aumento da vida útil do revestimento refratário do convertedor e maximização na produção de aço $[4,5]$.

A dessiliciação prévia do ferro-gusa pode ser feita no canal de corrida do alto forno, no carro torpedo ou na panela de transferência de gusa. No canal de corrida do alto forno geralmente utiliza-se misturas à base de cal e minério de ferro (carepa). Já no carro torpedo e na panela de transferência, além da injeção de mistura dessiliciante a base de $\mathrm{CaO}$ e $\mathrm{FeO}$, é comum também o sopro de gás oxigênio a fim de melhorar a eficiência do processo e manter as condições térmicas do metal.

Segundo Turkdogan [1], a injeção de gás oxigênio no ferro-gusa pode aumentar sua temperatura entre $120^{\circ} \mathrm{C}$ e $150^{\circ} \mathrm{C}$. Kawauchi et al [6] estudaram entre outros fenômenos, o efeito entre a variação de silício e a variação de temperatura do ferrogusa mediante utilização de fontes de oxigênio gasoso e sólido, neste estudo foi observado que a injeção de oxigênio gasoso ou a adição de carepa no ferro-gusa possui efeito similar no que tange a redução da concentração de silício no metal. Entretanto, para uma mesma variação de silício no ferro-gusa, a adição de carepa reduz a temperatura do banho metálico, enquanto que a injeção de oxigênio gasoso favorece o aumento da temperatura do metal. 
Além dos fatores termodinâmicos citados até aqui, a dessiliciação também é influenciada por fatores cinéticos. Durante o período inicial de oxidação do silício, geralmente a reação é controlada pelatransferência de massa na interface metalescória. A equação abaixo rege a cinética da reação de dessiliciação [7].

$$
-\frac{d[\% S i]}{d t}=\frac{A}{W_{m}} \cdot \frac{1}{\frac{L_{S i}^{*}}{k_{m} \cdot \rho_{m}}+\frac{1}{k_{S} \cdot p_{S}}} \cdot\left\{[\% S i] \cdot L_{S i}^{*}-(\% S i)\right\}
$$

Onde,

$\frac{d[\% S i]}{d t}$ : Taxa de Dessiliciação; $k$ : Coeficiente de transferência de massa $(\mathrm{m} / \mathrm{s}) ; m, s$ : Denotam a fase metal e a fase escória, respectivamente.; $\rho$ : Densidade do metal $\left(\mathrm{kg} / \mathrm{m}^{3}\right) . L_{S i}^{*}$ : Coeficiente de partição do silício entre o metal e a escória no equilíbrio; $(\% \mathrm{Si})$ : Concentração de silício na escória; [\%Si]:Concentração de silício no metal.; $A$ : Área Interfacial $\left(\mathrm{m}^{2}\right)$.; $W_{m}=$ Massa de metal $(\mathrm{kg})$.

Analisando a equação cinética da dessiliciação, observa-se que quanto maior a área interfacial entre a escória e o metal, maior será a taxa de dessiliciação. Além disso, escórias com maior densidade também favorecem a reação. Entretanto, a massa do metal desfavorece a mesma.

Nakasuga et al [8] avaliaram a dessiliciação no canal de corrida do alto forno n. 3 da planta de Kobe, comparando os métodos convencional (injeção) com um novo método proposto (agitação mecânica). A mistura utilizada foi $5 \%$ de $\mathrm{FeO}, 58 \%$ de $\mathrm{Fe}_{2} \mathrm{O}_{3}, 21 \%$ de $\mathrm{CaO}$ e $8 \%$ de $\mathrm{SiO}_{2}$, cuja taxa de injeção foi de $23,3 \mathrm{~kg} /$ ton de metal. Segundo os autores, a agitação mecânica afeta significativamente o processo de dessiliciação no canal de corrida, uma vez que se obtiveram concentrações menores de silício no ferro-gusa após o processo. Tal efeito ocorreu devido ao aumento do arraste da escória de topo para dentro do metal agitado.

\section{MATERIAIS E MÉTODOS}

\subsection{Definição das misturas dessiliciantes}

A definição da composição química e massa das misturas dessiliciantes foi feita a partir das equações 3 e 5. Com base nas equações de oxidação do silício através do $\mathrm{FeO}$ e consequente fixação do óxido formado pelo $\mathrm{CaO}$, foi calculada a massa estequiométrica de $\mathrm{FeO}$ necessária para reduzir a concentração de Si no ferro-gusa de $0,5 \%$ para $0,15 \%$, concentração última que, de acordo com vários autores $[4,5]$, contribui para que uma posterior etapa de desfosforação do ferro-gusa seja mais eficiente. Além disso, como $\mathrm{o} \mathrm{SiO}_{2}$ formado é fixado na escória pelo $\mathrm{CaO}$, em algumas misturas foi proposta a adição de $20 \%$ de fonte de $\mathrm{CaO}$ a mistura dessiliciante.

A fonte de $\mathrm{FeO}$ e $\mathrm{CaO}$ para a elaboração das misturas dessiliciantes foi fornecida pela Tecnosulfur, cuja composição química é mostrada na

Tabela 1.

Tabela 1. Composição química da fonte de $\mathrm{FeO}$ e $\mathrm{CaO}$ fornecida pela Tecnosulfur

\begin{tabular}{ccccc|cccc}
\hline \multicolumn{4}{c|}{ Fonte de FeO } & \multicolumn{4}{c}{ Fonte de CaO } \\
\hline \multicolumn{3}{c}{ Composição Química (\%) } & \multicolumn{3}{c}{ Composição Química (\%) } \\
\hline $\mathrm{Fe}_{2} \mathrm{O}_{3}$ & $\mathrm{SiO}_{2}$ & $\mathrm{Al}_{2} \mathrm{O}_{3}$ & $\mathrm{MnO}$ & $\mathrm{PPC}$ & $\mathrm{CaO}$ & $\mathrm{Al}_{2} \mathrm{O}_{3}$ & $\mathrm{MgO}$ & $\mathrm{PPC}$ \\
\hline 91,81 & 2,50 & 1,03 & 0,60 & 1,10 & 93,72 & 0,32 & 0,49 & 2,77 \\
\hline
\end{tabular}

PPC: Perdas por combustão. Fonte: Autor. 
Com base na composição química das fontes de $\mathrm{FeO}$ e $\mathrm{CaO}$ apresentadas na Tabela 1, foram propostas as seguintes misturas dessiliciantes.

- E1 - Adição da quantidade estequiométrica de fonte de FeO ao ferro-gusa;

- E2 - Adição de mistura contendo a quantidade estequiométrica de fonte de $\mathrm{FeO}$ e $20 \%$ de fonte de $\mathrm{CaO}$;

- D1 - Adição do dobro da quantidade estequiométrica de fonte de FeO ao ferro-gusa;

- D2 - Adição de mistura contendo o dobro da quantidade estequiométrica de fonte de $\mathrm{FeO}$ e $20 \%$ de fonte de $\mathrm{CaO}$.

As misturas D1 e D2 foram propostas com base no exposto por Campos [9], o qual cita que para se ter uma boa taxa de dessilciação, é necessário adicionar o dobro da massa da mistura calculada estequiometricamente. As misturas propostas foram resumidas na Tabela 2 .

Tabela 2. Composição química e massa das misturas dessiliciantes propostas.

\begin{tabular}{|c|c|c|c|c|c|c|c|c|c|}
\hline \multirow[b]{2}{*}{ Mistura } & \multicolumn{6}{|c|}{ Composição (\%) } & \multirow{2}{*}{$\begin{array}{c}\text { Massa da } \\
\text { fonte de } \\
\mathrm{FeO}(\mathrm{g})\end{array}$} & \multirow{2}{*}{$\begin{array}{c}\text { Massa da } \\
\text { fonte de } \\
\mathrm{CaO}(\mathrm{g})\end{array}$} & \multirow{2}{*}{$\begin{array}{c}\text { Massa da } \\
\text { Mistura } \\
\text { (g/kg de } \\
\text { gusa) }\end{array}$} \\
\hline & $\mathrm{Fe}_{2} \mathrm{O}_{3}$ & $\mathrm{CaO}$ & $\mathrm{SiO}_{2}$ & $\mathrm{Al}_{2} \mathrm{O}_{3}$ & $\mathrm{MgO}$ & MnO & & & \\
\hline E1 & 95,7 & - & 2,6 & 1,07 & - & 0,63 & 18,0 & - & 18,0 \\
\hline E2 & 76,5 & 19,7 & 2,2 & 0,97 & 0,12 & 0,51 & 18,0 & 4,5 & 22,5 \\
\hline D1 & 95,7 & - & 2,6 & 1,07 & - & 0,63 & 36,0 & - & 36,0 \\
\hline D2 & 76,5 & 19,7 & 2,2 & 0,97 & 0,12 & 0,51 & 36,0 & 9,0 & 45,0 \\
\hline
\end{tabular}

Fonte: Autor.

A composição química inicial do ferro-gusa ao quais as misturas dessiliciantes foram adicionadas é mostrada na Tabela 3.

Tabela 3. Composição química inicial do ferro-gusa usado nos testes experimentais e simulações termodinâmicas de dessiliciação.

\begin{tabular}{cccccccc}
\hline Mistura & Rotação & \multicolumn{5}{c}{ Composição Química (\%) } & \multirow{2}{*}{ Massa (kg) } \\
\cline { 5 - 7 } utilizada & (rpm) & C & Mn & Si & P & S & \\
\hline E1 & Não & 4,27 & 0,21 & 0,52 & 0,08 & 0,0025 & 1,0 \\
E2 & Não & 4,22 & 0,18 & 0,59 & 0,08 & 0,0033 & 1,0 \\
D1 & Não & 4,24 & 0,19 & 0,51 & 0,08 & 0,0036 & 1,0 \\
D2 & Não & 4,27 & 0,19 & 0,53 & 0,08 & 0,0026 & 1,0 \\
E1 & 500 & 4,27 & 0,21 & 0,52 & 0,08 & 0,0025 & 1,0 \\
E2 & 500 & 4,22 & 0,18 & 0,59 & 0,08 & 0,0033 & 1,0 \\
D1 & 500 & 4,24 & 0,19 & 0,51 & 0,08 & 0,0036 & 1,0 \\
D2 & 500 & 4,27 & 0,19 & 0,53 & 0,08 & 0,0026 & 1,0 \\
\hline
\end{tabular}

Fonte: Autor.

\subsection{Determinação das condições de equilíbrio das reações de Dessiliciação.}

A determinação das condições de equilíbrio das reações de dessiliciação foi realizada através do software FactSage. Para tanto, para a inserção das condições iniciais no software é necessário, além da composição química e massa das misturas, a massa e composição química do ferro-gusa.

De posse dos dados supracitados, foram selecionados os bancos de dados Fact53, FToxid e Fsstel para a determinação das condições de equilíbrio. Os bancos Fact53 e FToxid são utilizados para avaliar as fases formadas na escória na situação de 
equilíbrio e o banco Fsstel é utilizado para determinar a concentração de equilíbrio do ferro-gusa em contado com a mistura dessiliciante.

Entretanto, em cada banco de dados escolhido existe um sub-banco de dados que atua de forma a limitar as condições de equilíbrio do sistema em análise e incluir ou excluir alguns compostos que podem ou não estarem presentes na situação de equilíbrio termodinâmico. O sub-banco de dados para o Fact53 é O PureSolids, para o Ftoxid é o SlagA e para o Fsstel é o Liquid.

\subsection{Testes experimentais}

De posse dos resultados encontrados nos cálculos e simulações termodinâmicas, foram realizados os experimentos de dessiliciação do ferro-gusa. O procedimento adotado para as três etapas de pré-tratamento de ferro-gusa foi semelhante e, portanto, será abordada a seguir a forma geral de realização dos testes experimentais.

Inicialmente o forno elétrico modelo FE50RP cujo fabricante é a MAITEC LTDA foi programado para atingir a temperatura dos testes que é de $1400{ }^{\circ} \mathrm{C}$. Depois de atingida a temperatura do teste, o ferro-gusa sólido foi carregado em um cadinho de $\mathrm{MgO}-\mathrm{C}$, e colocado dentro do forno com o auxílio de uma tenaz. Após esse processo, gás argônio foi injetado na vazão de $10 \mathrm{NL} / \mathrm{min}$ a fim de inertizar a atmosfera e evitar a oxidação do metal. Depois de constatado que o ferro-gusa estava totalmente fundido, foi retirada uma amostra inicial do metal a fim de analisar a composição química inicial do mesmo. Em alguns experimentos, o banho foi agitado com a utilização de um impeler na rotação de 500 rpm, conforme Tabela 3. As amostras do metal foram retiradas com o auxílio de um amostrador a vácuo acoplado a uma haste de aço inoxidávelem intervalos de tempo de 0,1, 3, 5,7 ,10 e 15 minutos. A Figura 1 mostra um esquema da retirada de amostras durante os testes experimentais.

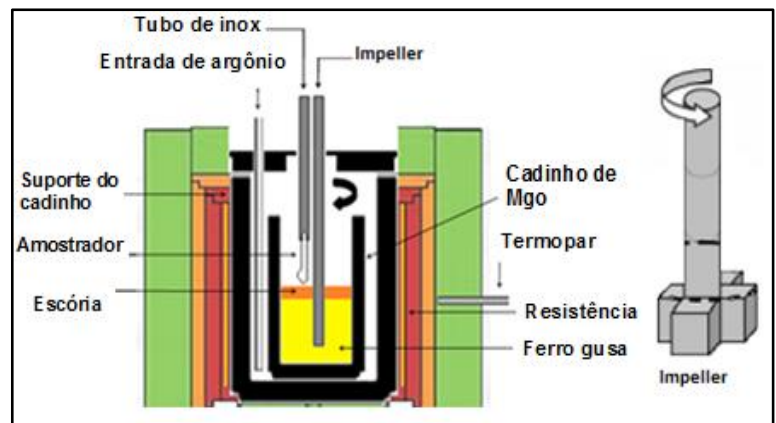

Figura 1. Figura esquemática da retirada de amostras durante os testes experimentais.

Depois de retirada a amostra inicial do metal, a mistura foi adicionada sobre o ferrogusa com o auxílio de um tubo de aço inoxidável com um funil acoplado em sua extremidade, a composição de cada mistura adicionada já foi apresentada anteriormente na Tabela 2.

\section{RESULTADOS E DISCUSSÃO}

\subsection{Resultados obtidos através da Termodinâmica Computacional}


Para determinar a redução do $\mathrm{Fe}_{2} \mathrm{O}_{3}$, foi realizada uma analise com uso do FactSage, onde a massa de $\mathrm{Fe}_{2} \mathrm{O}_{3}$ foi colocada em contato com $1 \mathrm{~kg}$ de ferro gusa à uma temperatura de $1400^{\circ} \mathrm{C}$, ao atingir o equilíbrio a mistura apresentou a total redução do $\mathrm{Fe}_{2} \mathrm{O}_{3}$ e, $\mathrm{FeO}$. Dessa forma, foi possível considerar que $0 \mathrm{Fe}_{2} \mathrm{O}_{3}$ presente nas misturas dessiliciantes é reduzido pelo Fe, conforme as equações a seguir [5].

$$
\begin{array}{r}
\left(\mathrm{Fe}_{2} \mathrm{O}_{3}\right)+[\mathrm{Fe}] \rightarrow 3(\mathrm{FeO}) \\
\Delta G^{0}=165,2-111,6 T\left(\frac{\mathrm{J}}{\mathrm{mol}}\right)
\end{array}
$$

Considerando essa situação, a composição química da mistura dessiliciante inserida no ThermoCalc a fim de avaliar a quantidade de líquido e sólido formado é a presente na Tabela 4.

Tabela 4. Composição química das misturas dessiliciantes após o contato com o banho

\begin{tabular}{|c|c|c|c|c|c|c|c|}
\hline \multirow{2}{*}{ Mistura } & \multicolumn{6}{|c|}{ Composição (\%) } & \multirow{2}{*}{$\begin{array}{c}\text { Massa da } \\
\text { Mistura (g) }\end{array}$} \\
\hline & $\mathrm{FeO}$ & $\mathrm{CaO}$ & $\mathrm{SiO}_{2}$ & $\mathrm{Al}_{2} \mathrm{O}_{3}$ & MgO & MnO & \\
\hline E1 & 96,8 & - & 1,95 & 0,80 & - & 0,45 & 18,0 \\
\hline E2 & 77,4 & 19,7 & 1,72 & 0,71 & 0,10 & 0,37 & 22,5 \\
\hline D1 & 96,8 & - & 1,95 & 0,80 & - & 0,45 & 36,0 \\
\hline D2 & 77,4 & 19,7 & 1,72 & 0,71 & 0,10 & 0,37 & 45,0 \\
\hline
\end{tabular}
metálico a $1400^{\circ} \mathrm{C}$.

A utilização de $20 \%$ de fonte de $\mathrm{CaO}$ nas misturas dessiliciantes E2 e D2 justifica-se através da Figura e Tabela 5, a qual o ponto A identifica as misturas E1 e D1, enquanto o ponto B mostra que as misturas E2 e D2.

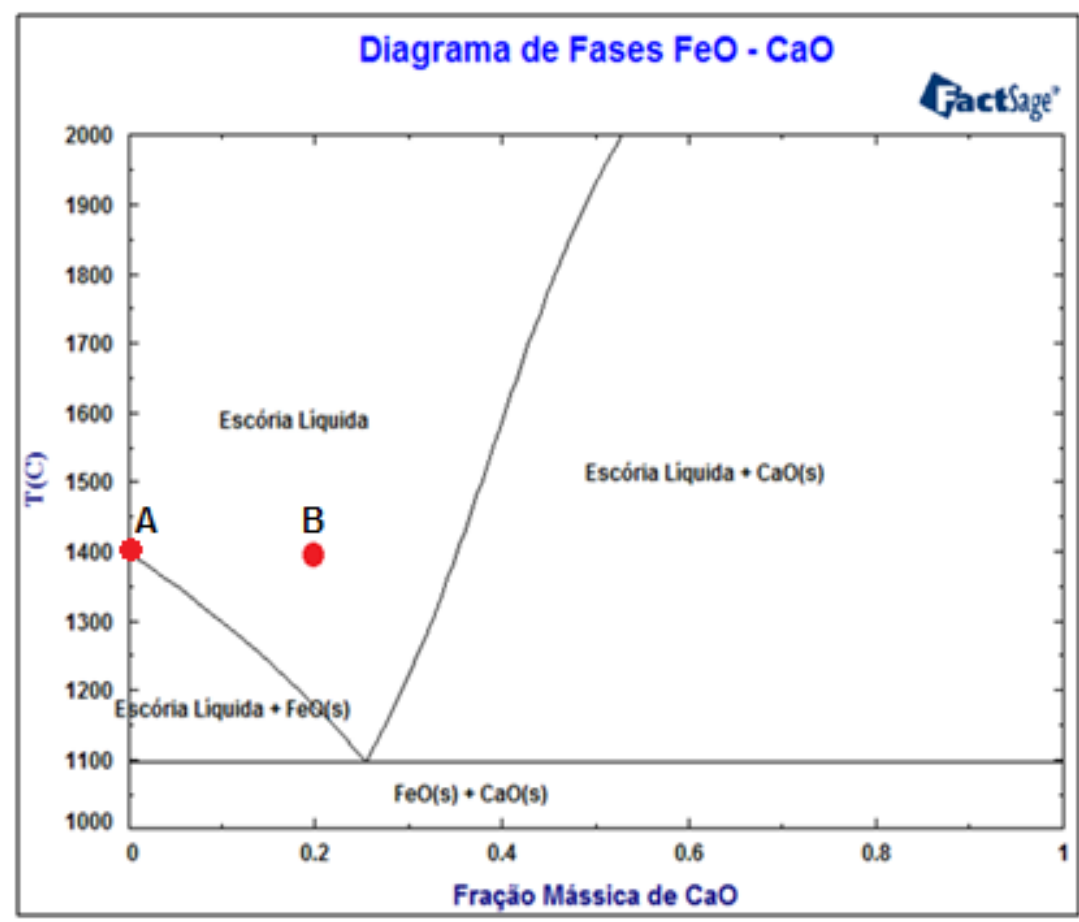

Figura 2. Diagrama de fases FeO-CaO obtido a partir do FactSage. Fonte: Autor.

A Tabela 5 mostra o resultado obtido através do ThermoCalc e confirma o fato exposto anteriormente. 
Tabela 5. Fases formadas na mistura dessiliciante a $1400{ }^{\circ} \mathrm{C}$.

\begin{tabular}{llcccc}
\hline \multirow{2}{*}{ Ponto } & \multirow{2}{*}{ Mistura } & \multirow{2}{*}{ \%Líquido } & Fases sólidas formadas & \multirow{2}{*}{ acao } & \multirow{2}{*}{ areo } \\
\cline { 3 - 5 } & & & \%FeO & & \\
\hline A & E1 e D1 & 75,0 & 25,0 & - & 1,0 \\
B & E2 e D2 & 100,0 & - & 0,54 & 0,8 \\
\hline
\end{tabular}

Fonte: Autor.

Observa-se através da Figura 2 e da Tabela 5, que as misturas E1 e D1 onde foi adicionado somente a fonte de FeO, apresentaram 25,1\% de fase sólida, sendo esta constituída somente de FeO. Já as misturas E2 e D2 contendo fonte de $\mathrm{FeO}$ e $20 \%$ de fonte de $\mathrm{CaO}$ apresentaram $100 \%$ de líquido a $1400{ }^{\circ} \mathrm{C}$, justificando que a adição de $\mathrm{CaO}$ aumenta a fração liquida.

Além da determinação das fases formadas nas misturas, também foram geradas curvas de aquecimento das mesmas, as quais são mostradas a seguir.
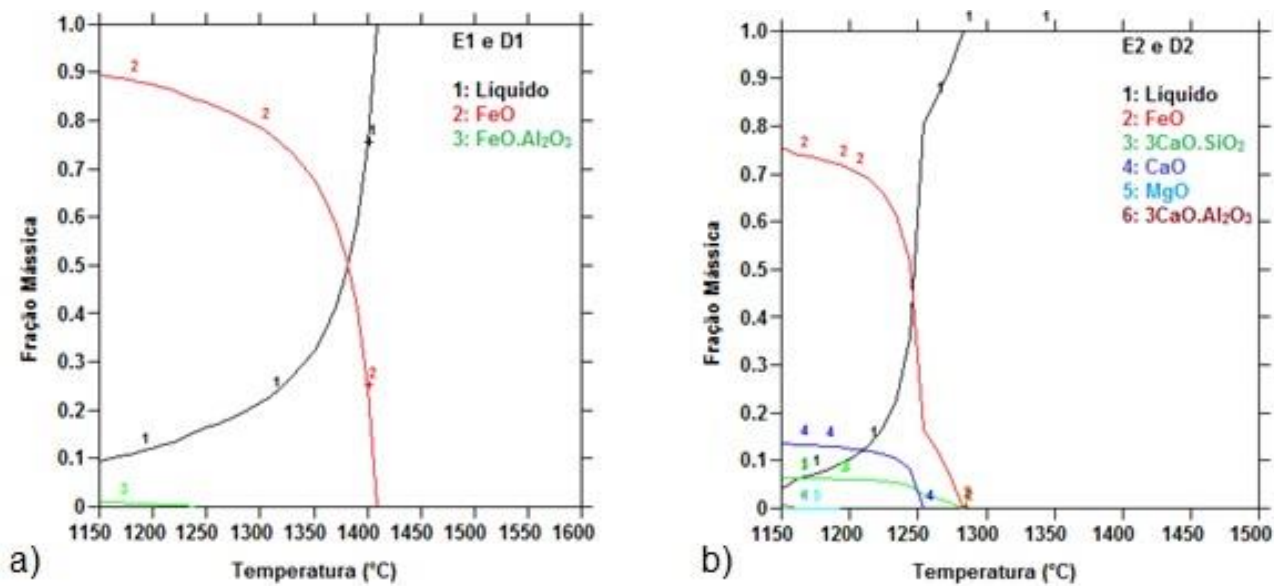

Figura 3. Curvas de aquecimento das misturas dessiliciantes obtidas através do ThermoCalc. a) Misturas com fonte de FeO. b) Misturas com fonte de $\mathrm{FeO}$ e $\mathrm{CaO}$. Fonte: Autor.

Observa-se a partir das curvas de aquecimento (Figura 3) que as misturas que são constituídas de apenas fonte de FeO (E1 e D1), possuem temperatura de fusão em torno de $1410^{\circ} \mathrm{C}$. Já as misturas contendo fonte de $\mathrm{FeO}$ e $\mathrm{CaO}$ (E2 e D2), estarão $100 \%$ líquidas a aproximadamente $1290^{\circ} \mathrm{C}$. De posse destes resultados, pode-se avaliar a importância das curvas de aquecimento geradas pelo ThermoCalc, uma vez que no caso das misturas $\mathrm{E} 1$ e $\mathrm{D} 1$, um aumento de $10^{\circ} \mathrm{C}$ na temperatura do processo resulta em um aumento na quantidade de líquido na mistura de $70 \%$ para $100 \%$. Já no caso das misturas E2 e D2, pode-se trabalhar com temperaturas inferiores a $1400{ }^{\circ} \mathrm{C}$ e, ainda assim, ter uma mistura com $100 \%$ de fase líquida.

Após determinada as fases formadas nas misturas, foram realizadas simulações termodinâmicas a partir do FactSage a fim de identificar as condições de equilíbrio do sistema, em especial, a concentração de equilíbrio do silício no ferro-gusa, a qual encontra-se em destaque na Tabela 6.

Tabela 6. Concentração de equilíbrio do ferro-gusa na etapa de dessiliciação a $1400{ }^{\circ} \mathrm{C}$.

\begin{tabular}{clllll}
\hline Mistura & {$\left[\%\right.$ Si $\left._{\text {eq. }}\right]$} & {$\left[\% \mathbf{O}_{\text {eq. }}\right]$} & {$\left[\% \mathbf{C}_{\text {eq }}\right]$} & {$\left[\% P_{\text {eq }}\right]$} & {$\left[\% S_{\text {eq }}\right]$} \\
\hline E1 & 0,178 & $2,21.10^{-4}$ & 4,27 & 0,08 & 0,0025 \\
E2 & 0,182 & $2,08.10^{-4}$ & 4,22 & 0,08 & 0,0019 \\
\hline
\end{tabular}




\begin{tabular}{llllll}
\hline D1 & $5,09.10^{-5}$ & $1,36.10^{-2}$ & 4,24 & 0,08 & 0,0036 \\
D2 & $1,12.10^{-5}$ & $1,32.10^{-2}$ & 4,27 & 0,07 & 0,0020 \\
\hline
\end{tabular}

Fonte: Autor.

Observa-se a partir da Tabela 6 que as misturas E1 e E2, as quais são relativas a adição da massa estequiométrica de $\mathrm{FeO}$ ao ferro-gusa, apresentaram redução na concentração de silício dentro do esperado, uma vez que os valores encontrados foram 0,178 e 0,182\%, respectivamente.Já as misturas nas quais foi adicionado o dobro da quantidade estequiométrica de FeO, D1 e D2, apresentaram como resultado uma alta redução na concentração de silício, 5,09.10-5\%Si eq e 1,12.10-5\% Sieq, respectivamente.

Além da concentração de equilíbrio do ferro-gusa, foi possível também identificar a concentração de equilíbrio da escória, bem como as fases formadas após a dessiliciação. Os resultados obtidos são mostrados na Tabela 7.

Tabela 7. Composição de equilíbrio da escória gerada no processo de dessiliciação $1400{ }^{\circ} \mathrm{C}$.

\begin{tabular}{ccc}
\hline \multirow{2}{*}{ Mistura } & \multirow{2}{*}{ \%Líquido } & Fases sólidas formadas (\%) \\
\cline { 3 - 3 } & & $\mathrm{SiO}_{2}$ \\
\hline E1 & 6,9 & 93,1 \\
E2 & 100,0 & - \\
D1 & 74,8 & 25,2 \\
D2 & 100,0 & - \\
\hline Fonte: Autor. & &
\end{tabular}

Observa-se a partir da Tabela 7 que nos casos em que foi adicionado $20 \%$ de $\mathrm{CaO}$ a mistura dessiliciante, a escória de equilíbrio continuou totalmente líquida. Já nos casos em que foram adicionadas as misturas contendo somente fonte de $\mathrm{FeO}$, parte do $\mathrm{SiO}_{2}$ formado na situação de equilíbrio estava sólido.

Portanto, pode-se inferir que a adição do $\mathrm{CaO}$, traz como benefício, principalmente, a fixação do $\mathrm{SiO}_{2}$ na escória e, tal fixação acarreta a diminuição do desgaste do revestimento refratário do equipamento destinado ao processo de dessiliciação, além de uma escória mais fluida, facilitando o processo difusional do silício desde o seio do banho metálico até a interface metal-escória.

\subsection{Resultados dos Testes Experimentais}

Conforme discutido na introdução, termodinamicamente, as misturas dessiliciantes possuem capacidade de reduzir a concentração do silício do ferro-gusa. Portanto, nesta seção serão abordados os resultados obtidos nos testes experimentais a fim de confirmar a análise supracitada e avaliar as variáveis cinéticas que influenciam na reação de dessiliciação.

A Tabela 8 mostra a concentração de silício inicial e final obtida nos testes experimentais de dessiliciação, bem como a eficiência alcançada. A eficiência dessiliciante foi calculada através da seguinte equação:

$$
\text { Rend. }(\%)=\frac{\left[\% S i_{i}\right]-\left[\% S i_{f}\right]}{\left[\% S i_{i}\right]} x 100
$$


Tabela 8. Concentração de silício inicial e final e eficiência obtida nos testes experimentais de dessiliciação a $1400^{\circ} \mathrm{C}$.

\begin{tabular}{ccccc}
\hline Mistura & Agitação $(\mathbf{r p m})$ & {$\left[\% \mathbf{S i}_{\mathbf{i}}\right]$} & {$\left[\% \mathbf{S i}_{\mathrm{F}}\right]$} & Eficiência (\%) \\
\hline E1 & Não & 0,596 & 0,512 & 14,0 \\
E2 & Não & 0,524 & 0,436 & 16,8 \\
D1 & Não & 0,539 & 0,413 & 23,3 \\
D2 & Não & 0,515 & 0,361 & 29,9 \\
E1 & 500 & 0,593 & 0,412 & 30,5 \\
E2 & 500 & 0,523 & 0,258 & 50,7 \\
D1 & 500 & 0,593 & 0,167 & 71,8 \\
D2 & 500 & 0,523 & 0,124 & 76,2 \\
\hline
\end{tabular}

Fonte: Autor.

Observa-se a partir da Tabela 8 que nenhuma das misturas dessiliciantes utilizadas no presente trabalho tiveram a capacidade de reduzir a concentração de silício no ferro-gusa para níveis abaixo de $0,15 \%$, conforme era esperado a partir das simulações termodinâmicas abordadas anteriormente.

A fim de se ter uma melhor visualização da eficiência dessiliciante obtido nos testes experimentais, o gráfico da

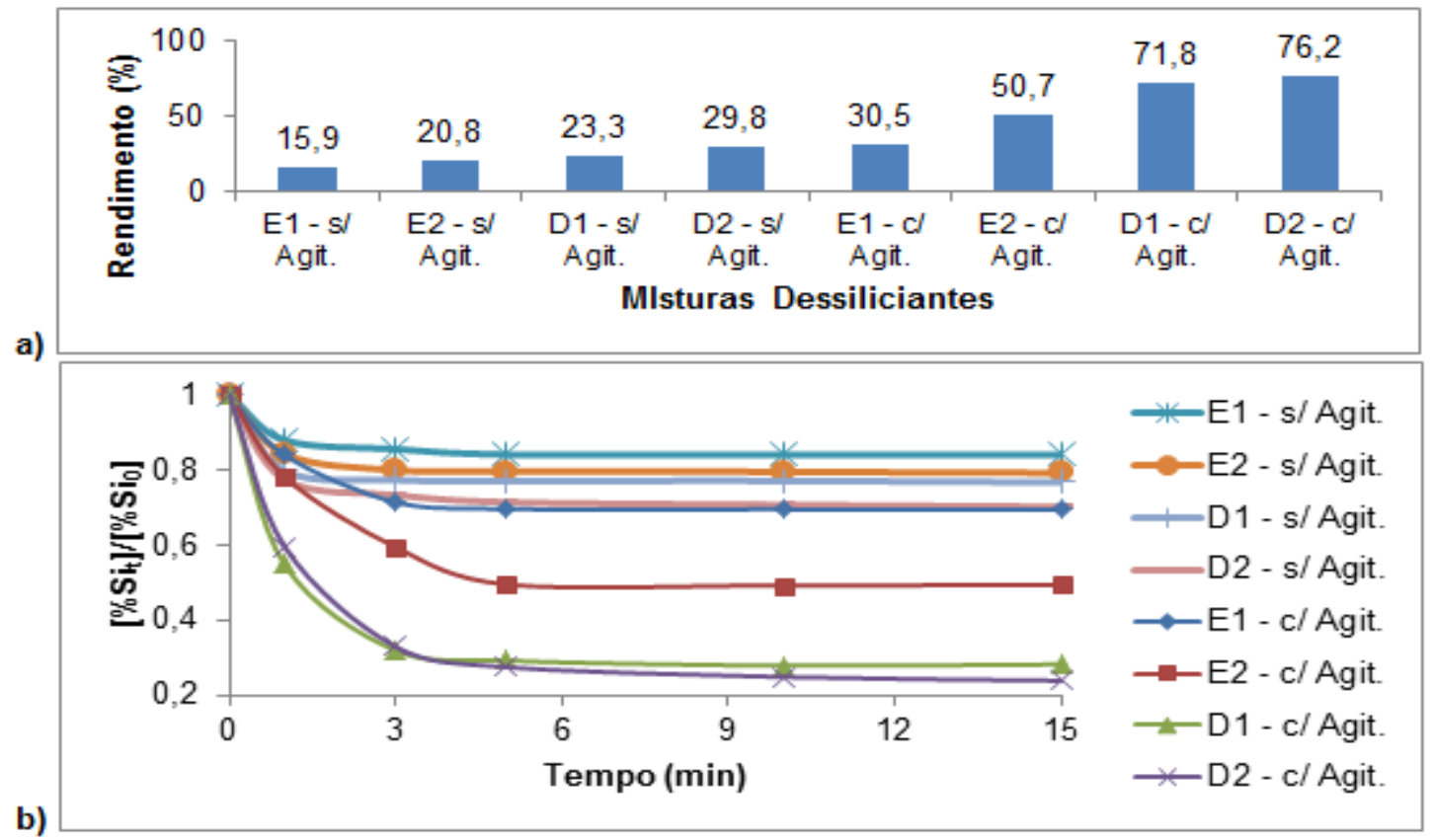

Figura (a) foi plotado com base nos dados presentes da Tabela 8, já para avaliar melhor as variáveis cinéticas do processo, segue a Figura 4(b), a qual mostra a variação da relação (\%Sit/\%Sio) no ferro-gusa mediante o tempo. Onde \%Sit é a concentração de silício em um tempo qualquer e \%Sio é a concentração de silício inicial. 
a)
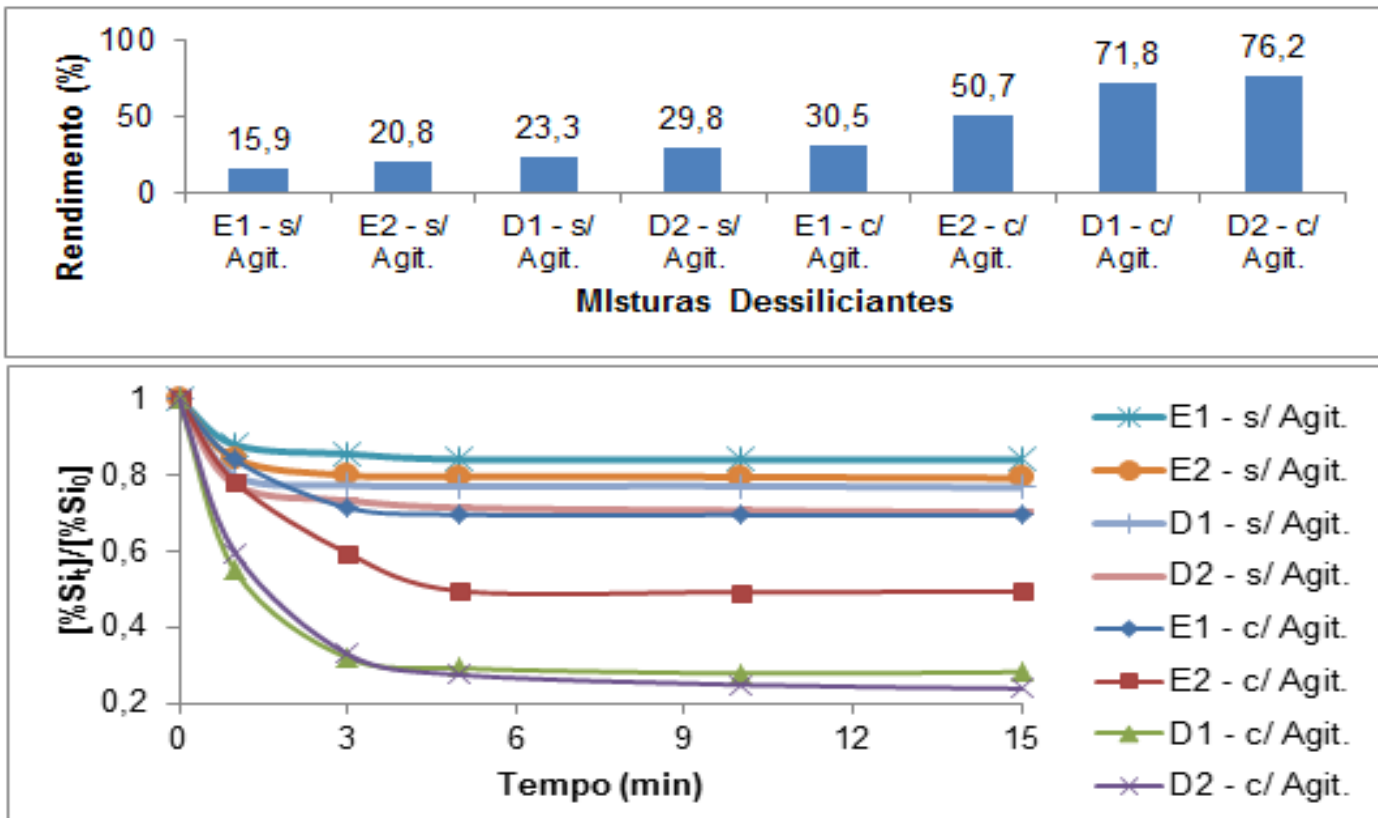

b)

Figura 4. (a) Rendimento obtido nos testes de dessiliciação do ferro-gusa a $1400^{\circ} \mathrm{C}$. (b) Variação da relação $\left(\% \mathrm{~S}_{t} / \% \mathrm{Si}_{i}\right)$ mediante o tempo nos testes de DeSi realizados a $1400{ }^{\circ} \mathrm{C}$. Fonte: Autor.

Observa-se na Figura 4 (a) que as misturas que apresentaram maior eficiência dessiliciante foram àquelas em que se empregou a agitação do banho metálico. Já entre as misturas onde não foi realizada a agitação, aquelas com o dobro da quantidade estequiométrica de fonte de $\mathrm{FeO}, \mathrm{D} 1 \mathrm{e} \mathrm{D2}$, obtiveram maior eficiência do que aquelas com a quantidade estequiométrica de fonte de $\mathrm{FeO}$, E1 e E2.

A Figura 4 (b) mostra que para todas as misturas empregadas no presente trabalho, a variação da concentração de silício seguiu um mesmo padrão, o qual foi a redução considerável da concentração de silício até o minuto 5 de experimento e, a partir daí não houve redução considerável da concentração do mesmo. Além disso, nota-se que a mistura que se destaca no que diz a respeito a maior taxa de dessiliciação é a mistura D2 com agitação. Tendo em vista este fato, a seguir são comparadas as misturas com e sem agitação.

No entanto, é válido lembrar que as misturas "E" são aquelas em que foi adicionada a quantidade estequiométrica de fonte de $\mathrm{FeO}$ e as misturas " $\mathrm{D}$ " as que foi adicionado o dobro da quantidade estequiométrica de fonte de FeO. Já o índice "1" indica que foi adicionada somente fonte de $\mathrm{FeO}$ e o índice "2" indica que foi adicionada fonte de $\mathrm{FeO}$ e $20 \%$ de fonte de $\mathrm{CaO}$ à mistura.

\subsection{Comparação da capacidade dessiliciante das misturas utilizadas sem agitação do sistema}

Observa-se a partir da 
a)

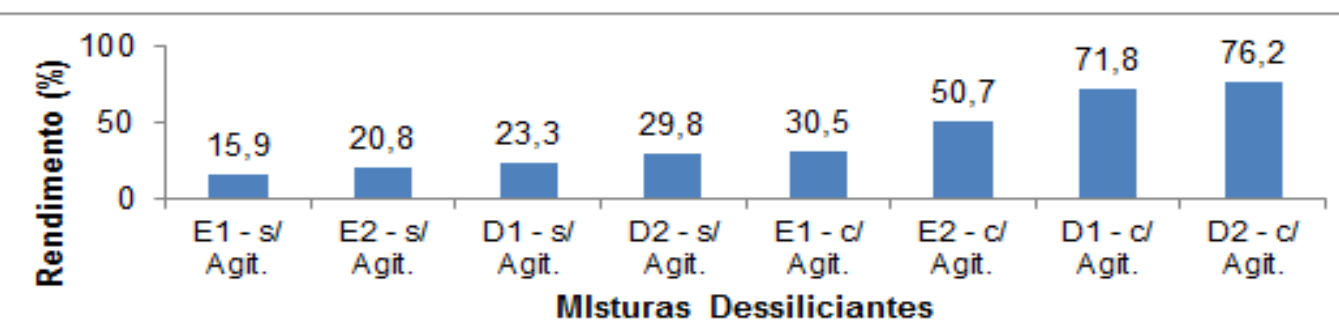

b)

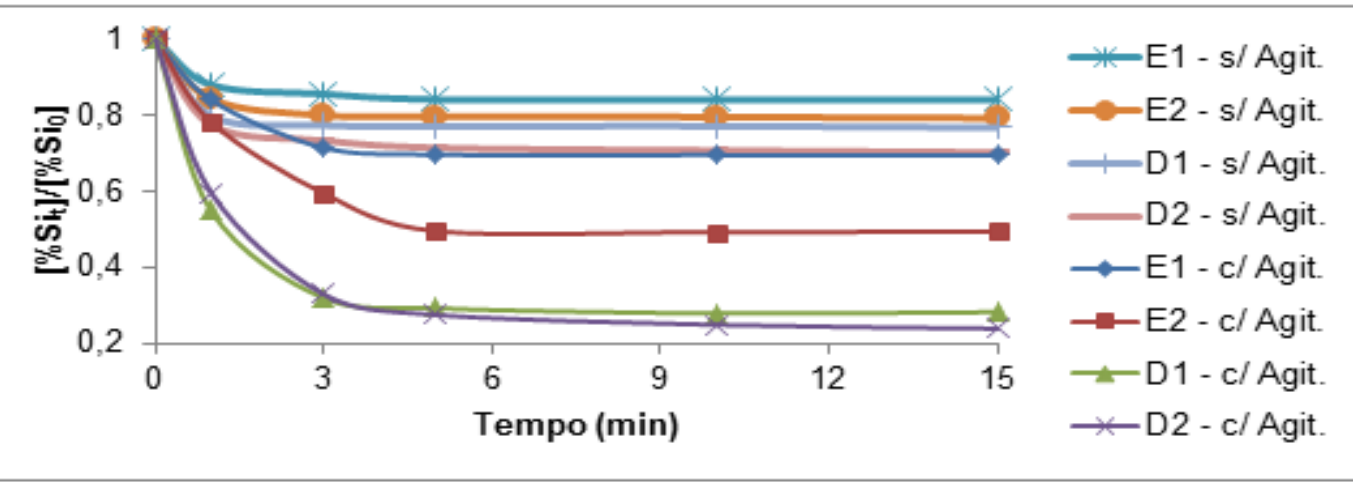

Figura (a) e da

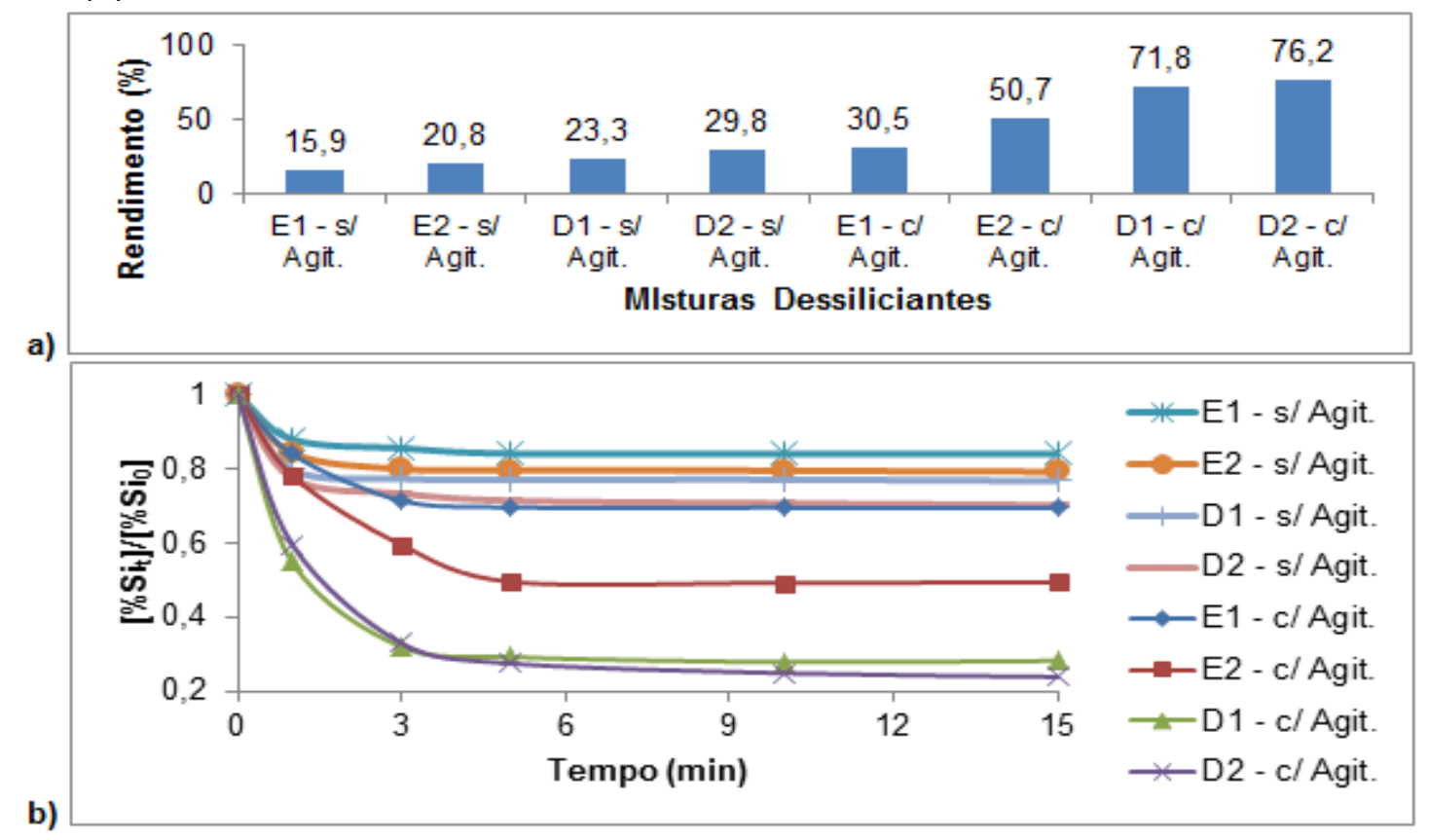

Figura (b) que as misturas E2 e D2 apresentaram maior taxa de dessiliciação e eficiência do que as misturas E1 e D1. Tal fato pode ser justificado pela adição de $20 \%$ de fonte de $\mathrm{CaO}$ às misturas $\mathrm{E} 2$ e $\mathrm{D} 2$, sendo que o $\mathrm{CaO}$ tem como finalidade fixar o $\mathrm{SiO}_{2}$ formado na escória, inibindo uma possível reversão do silício para o banho metálico, conforme encontrado por Yamagata et al [3] e apresentado na equação 11.

Erro! Fonte de referência não encontrada. $[\mathrm{Si}]+2(\mathrm{FeO})+3(\mathrm{CaO})=$

$$
\left(3 \mathrm{CaO} . \mathrm{SiO}_{2}\right)+2[\mathrm{Fe}]
$$

Também pode ser notado que a adição do dobro da quantidade estequiométrica de fonte de $\mathrm{FeO}$ às misturas contribui para o aumento da eficiência obtida nas mesmas, 
uma vez que tinha-se nessas misturas, D1 e D2, uma maior disponibilidade de oxigênio para a reação de oxidação do silício.

\subsection{Comparação da capacidade dessiliciante das misturas utilizadas com e sem agitação do sistema}

É possível observar mediante a

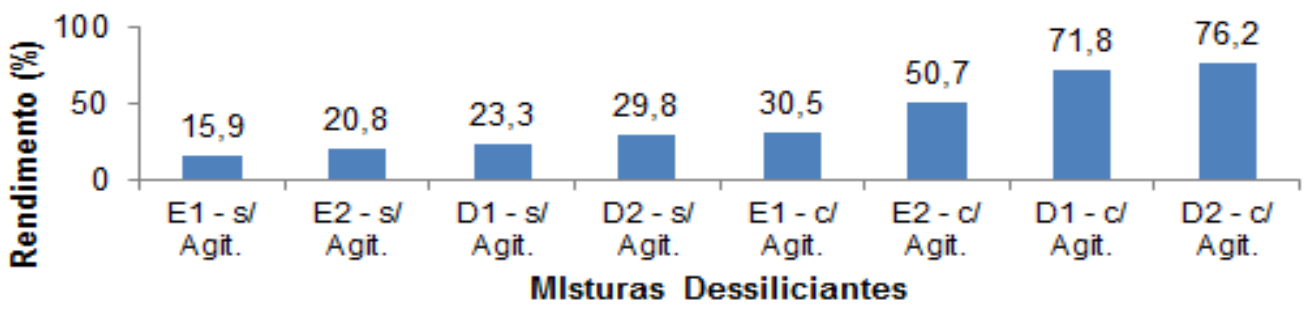

a)

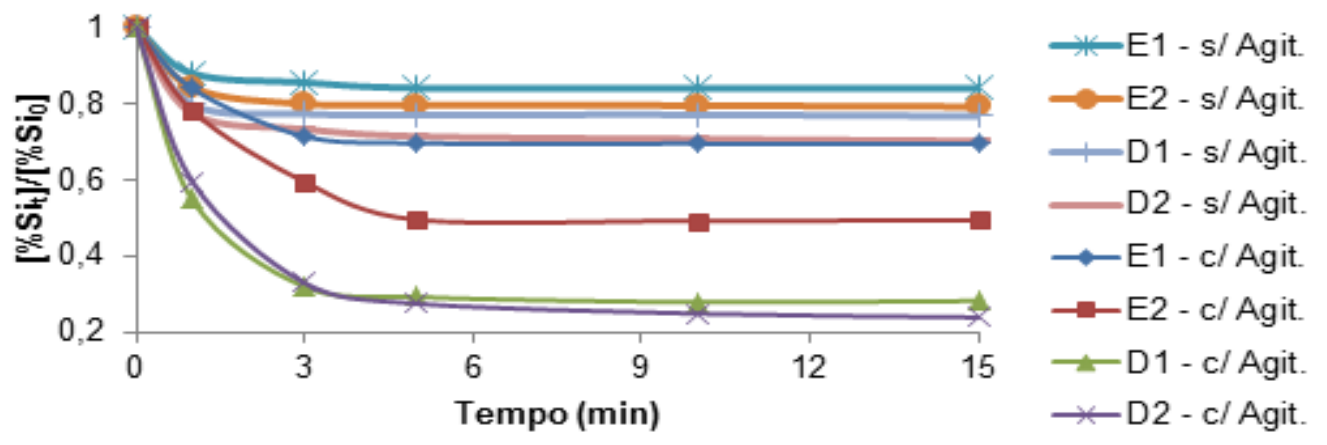

b)

Figura (a) e
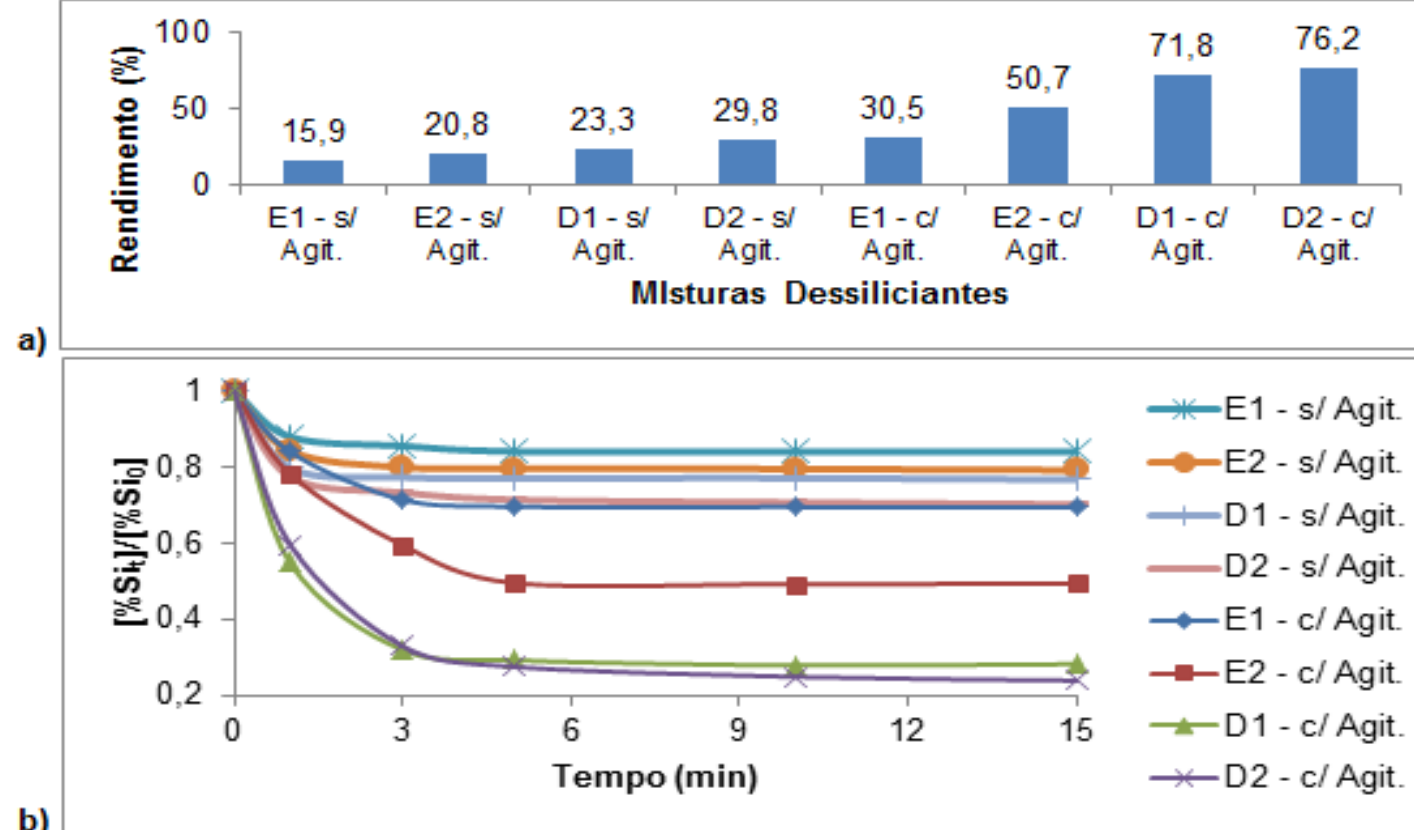

a)

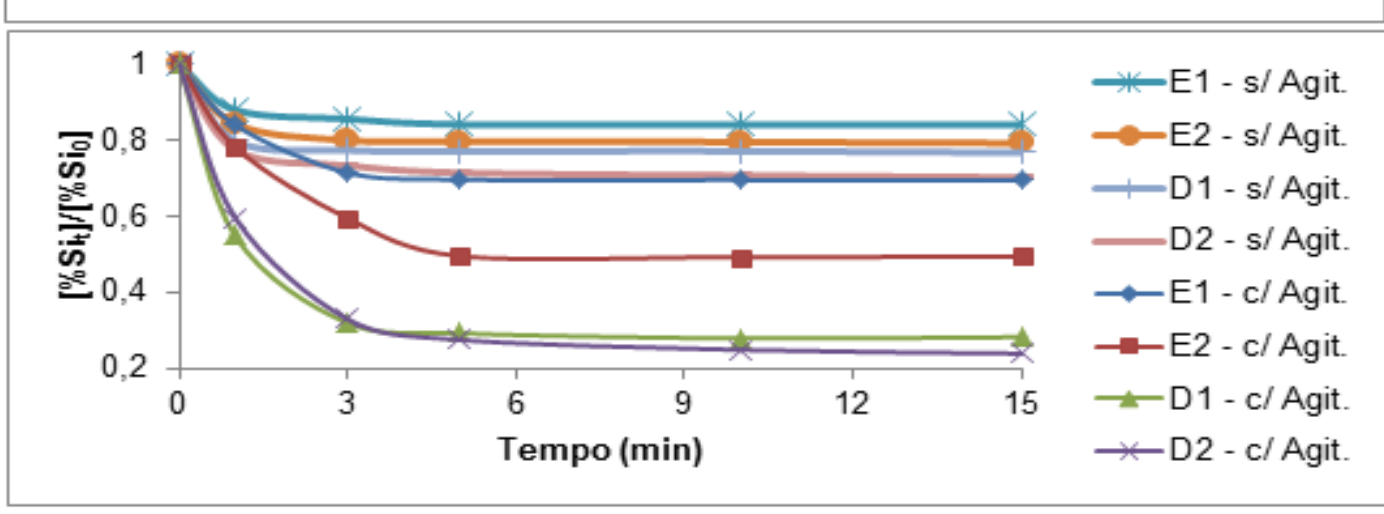

Figura (b) que a dessiliciação é fortemente influenciada pela agitação do sistema, uma vez que as misturas em que foi empregada a agitação apresentaram eficiência superior àquelas em que não foi empregada a agitação do sistema. Tal fato também foi encontrado por Nakasuga et al. [8]. Além disso, conforme abordado no item anterior, as misturas em que foi adicionado $20 \%$ de fonte de $\mathrm{CaO}$ apresentaram maior taxa de dessiliciação e eficiência. 
Portanto, pode-se concluir mediante os resultados experimentais que as misturas propostas E1, E2, D1 e D2 possuem capacidade de reduzir à concentração do silício no ferro-gusa, entretanto, a redução não foi àquela esperada a partir das simulações realizadas através dos softwares de termodinâmica computacional, a qual era reduzir tal concentração para níveis abaixo de $0,15 \%$. Além disso, as misturas E1 e E2, cujas quais foi empregada agitação de $500 \mathrm{rpm}$ apresentaram boas taxas de dessiliciação quando comparadas àquelas empregadas sem agitação, no entanto, as mesmas também não objetivaram a redução esperada para o presente trabalho.

\subsection{Comparação de Resultados}

Mediante os resultados termodinâmicos e experimentais pode-se avaliar a influência de alguns parâmetros sobre a eficiência da dessiliciação. Os resultados são mostrados na Tabela 9.

Tabela 9. Eficiência, característica das misturas iniciais e da escória gerada no equilíbrio termodinâmico dos testes de dessiliciação.

\begin{tabular}{cccccccc}
\hline Mistura & $\begin{array}{c}\text { Agitação } \\
\text { (rpm) }\end{array}$ & $\begin{array}{c}\text { Eficiência } \\
\text { (\%) }\end{array}$ & \%Si eq. & & \multicolumn{2}{c}{$\begin{array}{c}\text { Características da } \\
\text { Mistura Inicial }\end{array}$} & \multicolumn{2}{c}{$\begin{array}{c}\text { Características da } \\
\text { Escória } \\
\text { (Equilíbrio) }\end{array}$} \\
\cline { 5 - 8 } & & & & \%Líquido & $\begin{array}{c}\text { \%FeO } \\
\text { Sólido }\end{array}$ & \%Líquido & $\begin{array}{c}\text { \%SiO } \\
\text { Sólido }\end{array}$ \\
\hline E1 & Não & 14,0 & 0,178 & 74,9 & 25,1 & 6,9 & 93,1 \\
E2 & Não & 16,8 & 0,182 & 100,0 & - & 100 & - \\
D1 & Não & 23,3 & $5,09.10^{-5}$ & 74,9 & 25,1 & 74,8 & 25,2 \\
D2 & Não & 29,9 & $1,12.10^{-5}$ & 100,0 & - & 100 & - \\
E1 & 500 & 30,5 & 0,178 & 74,9 & 25,1 & 6,9 & 93,1 \\
E2 & 500 & 50,7 & 0,182 & 100,0 & - & 100 & - \\
D1 & 500 & 71,8 & $5,09.10^{-5}$ & 74,9 & 25,1 & 74,8 & 25,2 \\
D2 & 500 & 76,2 & $1,12.10^{-5}$ & 100,0 & - & 100 & - \\
\hline
\end{tabular}

Fonte: Autor.

A partir da Tabela 9 pode-se notar que a simulação a partir dos softwares de termodinâmica computacional identificou com relativa precisão quais seriam as misturas com maior capacidade dessiliciante, uma vez que os resultados encontrados para a concentração de silício de equilíbrio no ferro-gusa foram coerentes com a eficiência obtida nos testes experimentais quando não empregada agitação.

Além disso, pode-se observar que, comparativamente, as misturas identificadas com o índice "1" apresentaram menor eficiência dessiliciante em relação as misturas identificadas com o índice "2", e tal fato pode ser justificado pela quantidade de líquido e fases sólidas formadas tanto na mistura inicial quanto na escória de equilíbrio. Observa-se que as misturas "1" continham inicialmente 25,1\% de FeO sólido e, após o processo de dessiliciação, $\mathrm{o} \mathrm{SiO}_{2}$ gerado devido a reação de dessiliciação estava sólido, o que pode ter contribuído para uma limitação na difusão do silício do seio do banho até a interface metal-escória. Já as misturas "2" estavam totalmente líquidas tanto inicialmente quanto na situação de equilíbrio termodinâmico, o que contribui para maior mobilidade dos elementos envolvidos no processo de dessiliciação do ferro-gusa.

Portanto, pode-se obervar a partir do exposto acima que os softwares de termodinâmica computacional auxiliam tanto na simulação das condições de 
equilíbrio termodinâmico do processo quanto na identificação de variáveis que podem vir a influenciar cineticamente o processo de dessiliciação.

\section{CONCLUSÃo}

Depois de realizados os testes experimentais de dessiliciação e as simulações termodinâmicas computacionais, pôde-se chegar às seguintes conclusões:

- Os testes experimentais permitiram identificar que as misturas quando empregadas com a agitação do sistema mostraram-se mais eficazes no que tange a remoção do silício do ferro-gusa. Foi possível identificar também que quanto maior a massa de mistura adicionada ao ferro-gusa, maior a eficiência dessiliciante obtida;

- As misturas quando empregadas sem a agitação do sistema apresentaram uma redução média de $18 \%$ do silício no ferro-gusa no primeiro minuto de experimento e, a partir do $3^{\circ}$ minuto, a redução ocorreu de maneira suave com uma redução média de $1,35 \%$ de Si. Já as misturas onde foi aplicada a agitação de 500 rpm, as misturas apresentaram uma redução média de $34,6 \%$ da concentração de silício nos primeiros três minutos de experimento e, $0,29 \%$ a partir do $5^{\circ}$ minuto;

- Somente as misturas D1 e D2 foram capazes de reduzir a concentração de silício para níveis em torno de $0,15 \%$, o qual era o objetivo do presente trabalho. Um dos motivos das misturas E1 e E2 não terem atingido tal objetivo mesmo com agitação é o fato de as mesmas terem aderido a parede do cadinho, não participando efetivamente da reação de dessiliciação;

- As misturas com adição de $20 \%$ de fonte de CaO, E2 e D2, apresentaram maior eficiência do que as misturas $\mathrm{E} 1$ e $\mathrm{D} 1$, as quais continham somente fonte de $\mathrm{FeO}$. Este comportamento pode ser justificado, principalmente, pela presença do $\mathrm{CaO}$ na mistura, o qual tem como finalidade fixar $0 \mathrm{SiO}_{2}$ formado na escória, inibindo uma possível reversão do silício para o banho metálico.

\section{Agradecimentos}

Agradecimento ao IFES pela bolsa de estudos concedida e a estrutura para necessária para os testes experimentais e a CAPES pelo auxilio fornecido.

\section{REFERÊNCIAS}

1 Carvalho JLR, Assis PS, Camilo RD, Figueira RM, Campos VF. Dados Termodinâmicos para Metalurgistas. Belo Horizonte: Universidade Federal de Minas Gerais; 1977.

2 Turkdogan ET. Fundamentals of Steelmaking. 1. ed. London: The Institute of Materials; 1996.

3 Yamagata C, Kajiwara Y, Suyama S, Miyake T. Desiliconization Reaction of Pig Iron with High FeO Containing Blast Furnace Slag under Pressurized and Cokecoexisting Condition. ISIJ International. 1990;30(9): 740-474.

4 Xiang-MI Y, Ning-chuan Y, Ling W, Jiang, D. The Necessity of Hot Metal Desiliconization Process. Procedia Earth and Planetary Science. 2011;2: 116121.

5 Baer H, Baret DF, Blumenschein CD, Burgo JA, Buss WE, Calderon A, et al. The Making, Shaping and Treating of Steel: Steelmaking and Refining Volume. 11. ed. Pittsburgh: The AISE Steel Foundation; 1998. 
6 Kawauchi Y, Maede H, Kamisaka E, Satoh S, Inoue T, Naki M. Metallurgical Characteristics of Hot Metal Desiliconization by Injecting Gaseous Oxygen. ISIJ International. 1983;15: 1730-1737.

7 Ishikawa M. Analysis of Hot Metal Desiliconization Behavior in Converter Experiments by Coupled Reaction Model. ISIJ International. 2004;44(2):316325.

8 Nakasuga T, Kimura S, Mimura T, Tadai R, Ito K, Ono R. Development of a Continuous Desiliconization Process in the Hot Metal Runner of a Blast Furnace. Steel Research. 2009;80(3): 530-534.

9 Campos VF. Curso sobre Pré-refino de Ferro-Gusa. 1. ed. Belo Horizonte: Universidade Federal de Minas Gerais; 1984. 